\title{
Negative priming and stimulus-response compatibility
}

\author{
LING-PO SHIU \\ Chinese University of Hong Kong, Shatin, Hong Kong \\ and \\ SYLVAN KORNBLUM \\ University of Michigan, Ann Arbor, Michigan
}

\begin{abstract}
The subjects in this study made incongruent naming responses to words and pictures that were presented on alternate trials-(e.g., say "car" to BIKE). Their response time was longer if the correct response for the current trial was the name of the stimulus presented on the preceding trial, as compared with a control condition. These results suggest that the tendency to produce the (congruent) name of the stimulus is automatically activated and then inhibited. The "negative priming" effects appeared stronger for words where pictures were primes than for pictures where words were primes. The implications of these results for negative priming and stimulus-response compatibility are discussed.
\end{abstract}

We are often slow to respond to objects that we have recently ignored. For example, when a subject selects a target in the presence of a distractor (on the basis of color, location, and so on) reaction time (RT) in responding as to the target's identity is 10 to $50 \mathrm{msec}$ slower if the target was a distractor on the immediately preceding trial than if it was not (e.g., Tipper \& Cranston, 1985). This effect is called negative priming because presentation of a stimulus on two consecutive trials, prime and probe trials, respectively, causes interference on the probe trial.

Some researchers (e.g., Neill, 1977; Tipper, 1992) have argued that negative priming is evidence for the existence of inhibitory mechanisms in selective attention. They have hypothesized that unselected information is actively inhibited, and that this inhibition is carried over to the subsequent trial, thus delaying the response.

To produce negative priming, researchers have used tasks in which the prime trials contain a target and one or more distractors that are each associated with a different response. Although the distractor(s) may or may not cause observable interference in RT on the prime trial (Driver \& Tipper, 1989), it is assumed that conflict between a target and a distractor is necessary for the production of inhibition of the target. Furthermore, Moore (1994) has shown that negative priming effects may not occur if it is apparent that the probe trials do not contain a distractor that conflicts with the correct response (also see Lowe, 1979; Tipper \& Cranston, 1985). Thus, the presence of conflict in both

This research was supported by AFOSR Grant F49620-94-1-0020 to S.K. We thank Tram Neill, Hal Pashler, and Ed Smith for helpful comments on an earlier version of this manuscript, Tony Whipple for technical assistance, and Amrita Singh for help with data collection. Correspondence concerning this article should be addressed to Ling-Po Shiu, Department of Educational Psychology, Chinese University of Hong Kong, Shatin, Hong Kong (e-mail: lshiu@cuhk.edu.hk). prime and probe trials seems to be an important condition for producing negative priming effects (Fox, 1995; May, Kane, \& Hasher, 1995). In this study, we used a stimulusresponse compatibility (SRC) task to show that these two conditions can be met without the presence of distractors in the display, and that negative priming effects can be obtained in the absence of such distractors.

In their classic paper on SRC, Fitts and Deininger (1954) showed that given a stimulus and a response set that were related, some pairings of individual stimulus and response elements led to shorter RTs than did other pairings. For example, if a set of letters are used as stimuli, responses are faster and more accurate if each letter is paired with its own name as the response (congruent mapping) than if it is paired with the name of another letter (incongruent mapping). Kornblum (1994; Kornblum, Hasbroucq, \& Osman, 1990) has proposed the dimensional overlap model to account for this and other SRC effects. The model assumes that if a stimulus set and a response set are perceptually, conceptually, or structurally similar (i.e., have dimensional overlap), presentation of a stimulus element automatically activates its corresponding response. When the stimulusresponse mapping is incongruent, the automatically activated response conflicts with the response generated by a more controlled response-identification process. This conflict leads to an increase in response latency.

According to this view, even though there are no distractors in incongruent SRC tasks, selection has to be made between two conflicting response tendencies--one produced by a more automatic process and the other by a more controlled process. We propose that the inhibition mechanisms postulated in the standard negative priming tasks and the mechanisms that suppress the automatic response in incongruent SRC tasks might be the same, or at least have similar properties. If so, it implies that negative priming should occur when the unspoken name of the stimu- 
lus on a "prime" trial becomes the correct response on the following "probe" trial.

The present experiment put this hypothesis to test. Subjects were asked to respond to words or pictures (line drawings) with incongruent names (e.g., say "car" to BIKE). Words and pictures were shown on alternate trials. This permitted us to look at the priming effects of words and pictures. Previous studies of (positive) priming in naming tasks (e.g., Sperber, McCauley, Ragain, \& Weil, 1979) have suggested that pictures are more effective as primes and more susceptible to priming as targets than are words. Moreover, Tipper and Driver (1988) have argued that if negative priming can be found across modalities, it would imply that what is being inhibited is not merely a perceptual representation of the prime stimulus.

\section{METHOD}

\section{Subjects}

Twenty University of Michigan students were paid for their participation. All reported normal or corrected-to-normal vision and were right-handed, native English speakers between the ages of 19 and 25 .

\section{Design}

The subjects were divided into two groups, each with a different incongruent stimulus-response mapping. There were two within-subject factors: stimulus type and trial pair. Stimulus type was either a picture or a word. In a four-choice task, there were four possible types of trial pairs: (1) CR-CR-the correct response is repeated (e.g., "car" to BIKE followed by "car" to BIKE); (2) CR-S - the correct response for the prime trial becomes the probe stimulus (e.g., "bike" to BOAT followed by "car" to BIKE); (3) S-CR-the prime stimulus becomes the correct response for the probe trial (e.g., "plane" to CAR followed by "car" to BIKE); and (4) control-the stimulus and the correct response for the prime and probe trials are different (e.g., "boat" to plane followed by "car" to BIKE). ${ }^{1}$ Although we refer to a trial as a prime or probe trial, it should be noted that every trial, except for the first and last of each block, was a probe to the preceding trial and a prime to the succeeding trial.

\footnotetext{
Apparatus and Stimuli

The experiment was controlled by an IBM PC that generated stimulus displays and recorded responses in milliseconds. A vocal response triggered a voice key connected through an $\mathrm{A} / \mathrm{D}$ card to the computer. An automated speech-recognition device (Dragon Voice Tool) analyzed the speech input and identified it as one of four words in the vocabulary on which it had been trained. If the input did not match any of the words in the vocabulary satisfactorily, the device generated an "unintelligible" response. These trials were excluded from analysis.

The stimuli were four pictures and four words (Figure 1). They were presented in white against black on a CRT screen $(640 \times 480$ pixels $)$ From a viewing distance of $80 \mathrm{~cm}$, the pictures subtended visual angles from $6.24^{\circ} \times 7.13^{\circ}$ to $8.44^{\circ} \times 6.24^{\circ}$, and the words subtended visual angles from $2.68^{\circ} \times 0.89^{\circ}$ to $4.02^{\circ} \times 0.89^{\circ}$

\section{Procedure}

The subjects were divided into two groups, each with a different stimulus response mapping (Group 1: BIKE-"boat," BOAT-"plane," CAR-"bike," PLANE-"car"; Group 2: BIKE-"car," BOAT-"bike," CAR-"plane," PLANE"boat"). They were instructed to utter the response word appropriate to the target as quickly and as accurately as they could. Pictures and words were shown on alternate trials throughout a block, with pictures starting each block. All stimuli appeared with equal frequency in each block. The trial order was randomized and the four types of trial pairs were equally probable.

A pilot test indicated that the task was quite difficult at the beginning. So the subjects practiced for eight blocks of 40 trials each. Afterwards, they were tested for another eight blocks of 40 trials. The first 2 trials of
}

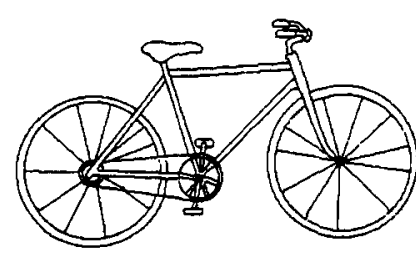

BIKE

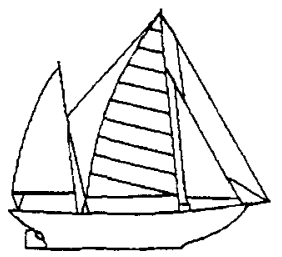

BOAT

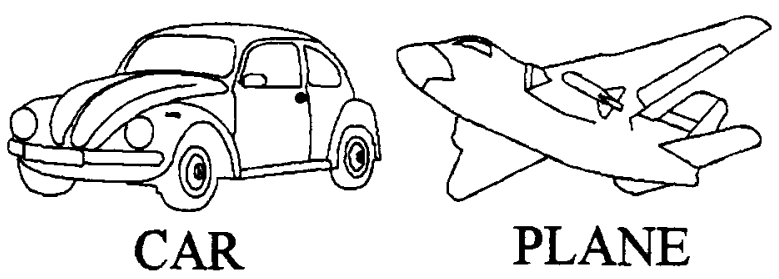

Figure 1. The pictures and words used in the experiment.

every block were discarded from analysis. A trial began with a tone $(750 \mathrm{~Hz}$ for $200 \mathrm{msec}$ ) and a plus sign in the middle of the CRT. Shortly after that $(700 \mathrm{msec})$, a stimulus appeared and remained visible until either a response was recorded or $2.5 \mathrm{sec}$ had elapsed. At the end of a trial, a feedback screen appeared for $1 \mathrm{sec}$. The intertrial interval was $2 \mathrm{sec}$.

\section{RESULTS}

Preliminary analysis indicated that mapping did not have any significant main or interaction effects. The two groups of subjects were therefore combined, and this factor was dropped from further analyses. The mean RT and error rates as a function of stimulus type and trial pair are shown in Table 1.2

RT was numerically longer for words ( $836 \mathrm{msec}$ ) than for pictures $(805 \mathrm{msec})$, but this difference was not significant $[F(1,19)=1.64, p>.1]$. The main effect of trial pair was significant $[F(3,57)=22.26, p<.001]$. The interaction of these two factors was marginally significant $[F(3,57)=2.16, p=.1]$. Planned comparisons were made to test the difference of each type of trial pair with control pairs. When the correct response was repeated (CRCR), RT was faster by about $100 \mathrm{msec}$ for both pictures and words $[F(1,19)=22.59, p<.001]$. The second type of trial pair (CR-S) did not differ significantly from control $[F(1,19)=1.19, p>.2]$. But the interaction with stimulus type was significant $[F(1,19)=11.9, p<.01]$. CR-S was faster than control for picture probes and slower than control for word probes. ${ }^{3}$ When a prime stimulus became a probe response (S-CR), RT was significantly slower than control $[F(1,19)=14.41, p<.01]$. In other words, there were negative priming effects. Word probes showed a numerically larger negative priming effect than did picture probes [ 43 and $11 \mathrm{msec} ; \mathrm{s}(19)=3.23$ and 1.97 , $p s<.01$ and .07 , respectively], although the difference was only marginally significant $[F(1,19)=3.42, p=.08]$. The error rates were low, and showed roughly the same patterns as RT. 
Table 1

Mean Response Time (RT) and Percent Error (\%E) on Probe Trials as a Function of Probe Stimulus and Trial Pair

\begin{tabular}{|c|c|c|c|c|c|c|c|c|c|c|}
\hline \multirow{3}{*}{$\begin{array}{c}\text { Probe } \\
\text { Stimulus }\end{array}$} & \multicolumn{8}{|c|}{ Trial Pair } & & \\
\hline & \multicolumn{2}{|c|}{$\begin{array}{l}\text { CR-CR } \\
\text { BIKE-"car" } \\
\text { BIKE-“car" }\end{array}$} & \multicolumn{2}{|c|}{$\begin{array}{c}\text { CR-S } \\
\text { BOAT-“"bike" } \\
\text { BIKE-"car"' }\end{array}$} & \multicolumn{2}{|c|}{$\begin{array}{c}\text { S-CR } \\
\text { CAR-"plane" } \\
\text { BIKE-"car" }\end{array}$} & \multicolumn{2}{|c|}{$\begin{array}{c}\text { Control } \\
\text { PLANE-"boat" } \\
\text { BIKE-“"car" }\end{array}$} & \multicolumn{2}{|c|}{$\begin{array}{l}\text { Negative } \\
\text { Priming }\end{array}$} \\
\hline & $\mathrm{RT}$ & $\% \mathrm{E}$ & RT & $\% \mathrm{E}$ & RT & $\% \mathrm{E}$ & RT & $\% \mathrm{E}$ & RT & $\% \mathrm{E}$ \\
\hline Pictures & 736 & 1.8 & 70 & 38 & 8 & 6 & 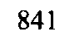 & 3 & $11^{*}$ & -0.3 \\
\hline Words & 757 & 3.0 & 856 & 4.0 & 884 & 5.3 & 841 & 4.3 & $43 \dagger$ & 1.0 \\
\hline$M$ & 746 & 2.4 & 825 & 3.9 & 868 & 5.6 & 841 & 5.3 & $27 \dagger$ & 0.3 \\
\hline
\end{tabular}

Note - CR, correct response; $S$, stimulus; negative priming is the difference between $\mathrm{S}-\mathrm{CR}$ and control. In this example, below each type of trial pair, the names of the stimuli are in small caps and the correct responses are in quotes. For each column pair, the prime stimulus is shown above the probe stimulus. ${ }^{*} p<.07 . \quad{ }^{\dagger} p<.01$.

\section{DISCUSSION}

We found that when subjects had to produce an incongruent naming response to a prime stimulus, the congruent response, or the natural name associated with that prime, became less accessible later when it was the required response to a different probe stimulus. The finding of such negative priming effects in an incongruent SRC task without distractors has important implications for the understanding of both SRC and negative priming. They are discussed next.

\section{Automatic Response Activation in SRC Tasks}

Fitts (1959) attributed the response delay in SRC tasks with incongruent mapping to a hypothetical stimulus recoding process. The dimensional overlap model proposed by Kornblum et al. (1990), on the other hand, suggests that incongruent RT is slow because it involves response conflicts between two opposing response tendencies: one activated by an automatic process and the other by a controlled process. Recent evidence from psychophysiological studies supports this view. Osman, Bashore, Coles, and Donchin (1992), for example, measured lateralized movement-related potentials (LRP) over the motor cortex while subjects responded to stimulus location (left or right) by moving a response lever on the opposite (incongruent) side. They found that LRP activation corresponding to the congruent but incorrect response emerged briefly before the activation corresponding to the correct response became dominant. Evidently, the congruent response was activated quickly and was eventually overwhelmed by the incongruent response. The occurrence of negative priming effects in our task provides converging behavioral evidence that the congruent response is activated even though the subjects tried to produce an incongruent response. Our results further imply that the congruent response is subsequently inhibited. The idea of activation followed by inhibition, in one variant or another, has been widely used to explain interference and negative priming effects caused by distractor stimuli irrelevant to a task (e.g., the Stroop effects) The present results suggest that this idea is also applicable to tasks in which interference and negative priming effects are caused by target stimuli alone.

Given that negative priming effects are found in an incongruent SRC task, the RT difference between congruent and incongruent mappings (also known as mapping effect) reported in the literature needs to be reexamined. Because no distractor stimuli are included in standard SRC tasks, negative priming cannot have occurred in the congruent condition. The difference between congruent and incongruent RT thus includes a negative priming component. ${ }^{4} \mathrm{O}$ course, negative priming cannot account for the entire mapping effect, because the former is usually much smaller than the latter.

\section{Negative Priming Without Distractors}

Our results show that even without distractors, covert response conflicts are sufficient to produce negative priming effects. These findings are easily explicable in terms of inhibitory mechanisms that suppress the congruent response on the prime trial. Yet they pose a serious chal- lenge to other hypotheses that have been proposed as a general account of negative priming. As several authors have pointed out (e.g., Neill \& Valdes, 1995), negative priming effects obtained in the standard paradigm with distractor stimuli are open to alternative explanations besides inhibition. In particular, the feature mismatch and the episodic retrieval hypotheses are often discussed (e.g., Fox, 1995). These two accounts, however, seem inadequate to explain our results. The feature-mismatch account argues that when a stimulus that was presented as a distractor on a prime trial reappears as a target on a probe trial, the stimulus changes value on one or more of the dimensions that define targets and distractors (e.g., from green to red), and this difference, which is a feature mismatch, causes interference. This explanation is not applicable to the present task simply because in the condition where negative priming effects occurred, the stimulus presented as target on a probe trial was not presented on the preceding prime trial. The episodic retrieval account assumes that a probe stimulus leads to implicit retrieval of information from the prime trial. The memory that this stimulus was ignored on the prime trial may interfere with selecting an appropriate response for the current trial. Although this account explains many results (see Neill, Valdes, Terry, \& Gorfein, 1992), it does not appear to be applicable to the present task either. In our task, on the probe trials where negative priming effects were found, the probe stimulus had not been presented, nor had its name been the correct response on the preceding prime trial. It is hard to see how the probe stimulus could have led to retrieval of information related to the prime trial. One could argue that when the correct response for the probe trial is identified, it leads to the memory that this response was inappropriate a few moments ago. But this is a significant departure from the original theory.

If the inhibition hypothesis is the most reasonable explanation of our results, it raises the question of what is being inhibited. We believe that what needs to be inhibited is the tendency to produce the natural name of the stimulus (which happens to be one of the response alternatives). Assuming that this tendency is likely to be more strongly activated for words than for pictures, one might expect that the former would lead to stronger inhibition and hence larger negative priming effects than would the latter. However, our results show larger negative priming effects with picture primes and word probes than the other way around. This could mean either that pictures are more effective primes or that words are more susceptible to negative priming. Unfortunately, the present design does not permit these two alternatives to be disentangled, because stimulus type (picture or word) on a prime trial is confounded with the opposite stimulus type on the probe trial. A follow-up experiment that manipulates stimulus type on prime and probe trials independently is currently under way.

\section{Negative Priming of Inappropriate Meanings}

Previous attempts have sought negative priming effects without distractors in tasks in which the stimuli have conflicting meanings. The conjecture was that presentation of an ambiguous word (e.g., BANK) activates several meanings associated with the word, but only one of those meanings is eventually selected on the basis of sentence context or other factors. The unselected meanings may then be less accessible when they 
become appropriate a few moments later. One of the earliest studies related to this question was conducted by Schvaneveldt, Meyer, and Becker (1976). They presented subjects with a sequence of three words in which the middle word (e.g., BANK) was a homograph whose meaning was biased by the first word (e.g., RIVER). The final target word in the sequence was related either to the meaning of the homograph defined by the first word (e.g., sTREAM) or to another meaning of the homograph (e.g., MONEY). Schvaneveldt et al. found that lexical decision time was faster to STREAM than to an unrelated control word. However, decision time to MONEY was not slower than the control. Hence, there was no evidence that the meaning of BANK as a financial institution was inhibited after RIVER and BANK had been presented (also see Simpson \& Burgess, 1985).

However, more recent studies (e.g., Hekkanen, 1981, cited in Neill, 1989; Simpson \& Kang, 1994) were able to find evidence of negative priming using homographs. Simpson and Kang presented a homograph (e.g., BANK) as a prime, followed after $200 \mathrm{msec}$ by a target word for a naming response. The target word was either related (e.g., STREAM) or unrelated to the homograph. After some intervening trials, the same homograph was presented as prime again. On this presentation, the target word that followed might be related to a second meaning of the homograph (e.g., MONEY). Simpson and Kang found that naming response was about $10 \mathrm{msec}$ slower to MONEY than to a control word that was not an associate of BANK. They concluded that the context-inappropriate meaning was less accessible than baseline level.

Aside from using a different task (naming vs. lexical decision), Simpson and Kang's (1994) study differed in another significant way from studies that had failed to find negative priming of context-inappropriate meaning (e.g., Schvaneveldt et al., 1976; Simpson \& Burgess, 1985). In their experiments, a homograph was presented as prime twice. It seems likely that presenting the homograph a second time may lead to retrieval of its meaning from the prior presentation. If this is, indeed, the case, then their results may best be explained by the episodic retrieval hypothesis. In contrast, our finding of negative priming effects with response conflicts alone is more in line with the inhibition hypothesis.

\section{Further Implications}

Incongruent SRC tasks have been used to test patients with frontal lobe lesions (e.g., Luria, 1973) as well as young children whose frontal lobes have not fully matured. Several authors (e.g., Diamond, 1990) have proposed that the dorsolateral prefrontal cortex is involved in controlling action through inhibition. Gerstadt, Hong, and Diamond (1994), for example, found that children below 5 years of age had much difficulty when asked to say "night" to a white card with a drawing of a brightly colored sun and to say "day" to a black card with a moon and stars. They argued that the task was difficult because it required inhibition of a natural response. Our results are consistent with their suggestion and provide evidence that inhibition affects the response on the successive trial

Subjects in incongruent SRC tasks are asked to respond to familiar stimuli with arbitrary and unnatural responses. This usually involves learning new associations on top of old ones, unless the SR mapping can be summarized by some simple rules (Duncan, 1977). From this perspective, the task resembles the A-B/A-C paradigm in the verbal learning literature. A long-standing unresolved question in that literature is whether or not learning new associations involves unlearning old ones A related question is whether the unlearning of old associations takes place during extinction. Although the present study was not intended as an investigation of that issue, it may have some bearing on it. One could argue that as long as negative priming effects are observed, the congruent response (which is the result of years of learning) is automatically activated every time the stimulus is presented. We divided the experimental trials in two and looked at negative priming effects in each half The effect was 11 and $14 \mathrm{msec}$ for picture trials and 40 and $46 \mathrm{msec}$ for word trials, for the first and second half, respectively. Because the negative priming effects did not get smaller with practice, it seems that when new associations are acquired, the old associations are inhibited but not unlearned (see Rescorla, 1993). Further studies that track the development of negative priming with more extended practice may lead to an answer to the question of unlearning.

\section{Conclusion}

Stimulus-response compatibility and selective attention are often treated as two separate areas of research, even though similar tasks have been studied in both areas (e.g., the Stroop task). The present study demonstrates a fruitful dialogue between the two areas. We have shown that negative priming effects occur in an incongruent SRC task. This supports the view that when people are required to make an incongruent response, the congruent response is still activated. By demonstrating the existence of negative priming effects in the absence of distractors, we have also provided support for the inhibition theory of negative priming.

\section{REFERENCES}

DiAMOND, A. (ED.) (1990). The development and neural bases of higher cognitive functions. (Annals of the New York Ácademy of Sciences, Vol. 608). New York: New York Academy of Sciences.

DRIVER, J., \& TIPPER, S. P. (1989). On the nonselectivity of "selective" seeing: Contrasts between interference and priming in selective attention. Journal of Experimental Psychology: Human Perception \& Performance, 15, 304-314.

DUNCAN, J. (1977). Response selection rules in spatial choice reaction tasks. In S. Dornic (Ed.), Attention and performance VI (pp. 49-61). Hillsdale, NJ: Erlbaum.

FitTs, P. M. (1959). Human information handling in speeded tasks (Report No. RC-109). Yorktown Heights, NY: IBM Research Center.

FitTs, P. M., \& DEININGER, R. L. (1954). S-R compatibility: Correspondence among paired elements within stimulus and response codes. Journal of Experimental Psychology, 48, 483-492.

Fox, E. (1995). Negative priming from ignored distractors in visual selection: A review. Psychonomic Bulletin \& Review, 2, 145-173.

Gerstadt, C. L., Hong, Y. J., \& Diamond, A. (1994). The relationship between cognition and action: Performance of children $3^{1 / 2-7}$ years old on a Stroop-like day-night test. Cognition, 53, 129-153.

Kornblum, S. (1994). The way irrelevant dimensions are processed depends on what they overlap with: The case of Stroop- and Simon-like stimuli. Psychological Research, 56, 130-135.

Kornblum, S., HasbroucQ, T., \& Osman, A. (1990). Dimensional overlap: Cognitive basis for stimulus-response compatibility-A model and taxonomy. Psychological Review, 97, 253-270.

Kornblum, S., \& LeE, J.-W. (1995). Stimulus-response compatibility with relevant and irrelevant stimulus dimensions that do and do not overlap with the response. Journal of Experimental Psychology: Human Perception \& Performance, 21, 855-875.

LOWE, G. (1979). Strategies, context, and the mechanism of response inhibition. Memory \& Cognition, 13, 74-80

LURIA, A. R. (1973). Higher cortical functions in man. New York: Basic Books.

MaY, C. P., Kane, M. J., \& Hasher, L. (1995). Determinants of negative priming. Psychological Bulletin, 118, 35-54.

MoORE, C. M. (1994). Negative priming depends on probe-trial conflict: Where has all the inhibition gone? Perception \& Psychophysics, 56, 133-147.

NEILL, W. T. (1977). Inhibitory and facilitatory processes in selective attention. Journal of Experimental Psychology: Human Perception \& Performance, $3,444-450$

NEILL, W. T. (1978). Decision processes in selective attention: Response priming in the Stroop color-word task. Perception \& Psychophysics, 23, 80-84.

NeILL, W. T. (1989). Lexical ambiguity and context: An activationsuppression model. In D. S. Gorfein (Ed.), Resolving semantic ambiguity (pp. 63-83). New York: Springer-Verlag.

NeILL, W. T., \& VALDES, L. A. (1995). Facilitatory and inhibitory aspects of attention. In A. F. Kramer, M. Coles, \& G. D. Logan (Eds.), Converging operations in the study of visual selective attention (pp. 77-106). Washington, DC: American Psychological Association.

Neil., W. T., Valdes, L. A., Terry, K. M., \& Gorfein, D. S. (1992). Persistence of negative priming: II. Evidence for episodic trace retrieval. Journal of Experimental Psychology: Human Perception \& Performance, 18, 993-1000.

Osman, A., Bashore, T. R., Coles, M. G., \& Donchin, E. (1992). On the transmission of partial information: Inferences from movementrelated brain potentials. Journal of Experimental Psychology: Human Perception \& Performance, 18, 217-232.

ResCorla, A. R. (1993). Inhibitory associations between S and R extinction. Animal Learning \& Behavior, 21, 327-336. 
Schyaneveldt, R. W., Meyer, D. E., \& Becker, C. A. (1976). Lexical ambiguity, semantic context, and visual word recognition. Journal of Experimental Psychology: Human Perception \& Performance, 2, 243-256.

Simpson, G. B., \& Burgess, C. (1985). Activation and selection processes in the recognition of ambiguous words. Journal of $E x$ perimental Psychology: Human Perception \& Performance, 11, 2839.

Simpson, G. B., \& KanG, H. (1994). Inhibitory processes in the recognition of homograph meanings. In D. Dagenbach \& T. H. Carr (Eds.), Inhibitory process in attention, memory, and language (pp. 359-381). San Diego: Academic Press.

SPerber, R. D., McCauley, C., Ragain, R. D., \& Weil, C. M. (1979) Semantic priming effects on picture and word processing. Memory \& Cognition, 7, 339-345.

TIPPER, S. P. (1992). Selection for action: The role of inhibitory mechanism. Current Directions in Psychological Science, 1, 105-109.

TIPPER, S. P., \& Cranston, M. (1985). Selective attention and priming: Inhibitory and facilitatory effects of ignored primes. Quarterly Journal of Experimental Psychology, 37A, 591-611.

TIPPER, S. P., \& DrIVER, J. (1988). Negative priming between pictures and words in a selective attention task: Evidence for semantic processing of ignored stimuli, Memory \& Cognition, 16, 64-70.

\section{NOTES}

1. For the sake of convenience, we wrote "the prime stimulus became the correct probe response," although a prime stimulus was either a picture or a word, whereas a correct response was the name of a picture or a word.

2. Reaction time for the erroneous trials as well as the trials immediately following them were excluded from analysis. The remaining data were then trimmed using the following procedure. First, we truncated the top and bottom $10 \%$ of the RT distribution for each condition by subject. On the basis of the mean and the standard deviation of this truncated set, we included all the data points of the data set before truncation that were within 3.3 SDs from the mean.

3. An analogue of this type of trial pair can be found in a standard negative priming task when a prime target (hence the correct response) becomes a probe distractor. Reaction time for these trials is sometimes faster than control (e.g., Neill, 1978). Our results show a more complicated pattern. Because this is not the focus of the paper, we leave it for further research.

4. For example, Kornblum and Lee (1995) investigated the speed of naming responses to letter stimuli and keypress responses to finger icons, with congruent and incongruent mapping. When their data from the incongruent conditions were reanalyzed for negative priming effects, we found an average effect size of 5 to $23 \mathrm{msec}$, depending on the condition.

(Manuscript received February 8, 1996; revision accepted for publication May 7, 1996.) 